are in progress, but it is believed that even if aphids are incriminated as vectors, they can only be regarded as of secondary importance in the spread of spike, as they are very rare on sandal. Moreover, aphids appear to be peculiarly fitted for the transmission of virus diseases under experimental conditions ${ }^{6}$.

These satisfactory results are the outcome of collaboration between several research organisations with the financial support of their respective governments. They have now to be translated into practical sylviculture, and it is hoped that the forest departments interested will be able to allot the additional funds required for this purpose.

\footnotetext{
2 Mycol. Ser., Dept. Agric., Mysore, Bull. 3; 1917.

Indian Forest Rec., 17, pt. I; 1932.

s ibid., 17, pt. ix.

- Quanjer, Phytopatholozy, 21; 1931.

${ }^{5}$ Bull., Boyce Thompson Institute, 5, pt. i ; 1933.

- Smith, Biol. Rev., 6; 1931.
}

\title{
X-Ray Analysis of Fibres
}

"P RESEN'T methods can still go far, but I am convinced that progress ... is about to gain a great impetus from the application of those new methods of research which chemistry is inheriting from physics: X-ray analysis . . ."* So spake in general terms the president of the British Association in his address on the evening of September 6: on the following morning, Sections A (Mathematical and Physical Sciences) and $\mathrm{K}$ (Botany) foregathered to demonstrate the point in somewhat more detail. That physicists should hobnob with botanists-and not simply for the purpose of drinking tea-and on the following Mondayt even be invited into the stronghold of vitalistically-minded zoologists is a very definite cause for congratulation, in spite of the dark mutterings of some that it is all very well to talk about the structure of molecules and adopt such an attitude of pitiable optimism in the face of 'life' and all the tremendous tale of the activities of living organisms !

In opening the joint discussion of Sections A and $\mathrm{K}, \mathrm{Mr}$. W. T. Astbury outlined some developments in the X-ray interpretation of the properties of hair, feathers and other protein structures. Recent progress in our knowledge of the molecular structure of natural fibres arises largely out of the recognition by $\mathrm{X}$-ray means that the solid state of fibres is a crystalline state, generally imperfect, it is true, yet nevertheless sufficiently organised to give valuable information about the form and properties of the giant molecules which orthodox chemistry suggests as their basis. The crystallites are sub-microscopic, but it can be seen at once from X-ray photographs that they are always effectively long and thin and lie with their long axes either roughly parallel to the fibre axis, as in silk and hair, or arranged spirally round it, as in ramie and cotton.

In the light of a mass of experimental evidence of one sort and another-and it must be emphasised that the study of the fine structure of biological subjects has advanced and will continue to advance only through a close alliance between all the various methods of attack - the conclusion seems irresistible that these crystallites, or organised aggregates, which make up the body of the fibre

* "Some Chemical Aspects of Life" (see NAture, Sept. 9, p. 389) $\uparrow$ Discussion on the structure of protoplasm, Section D, Sept. 11. substance and which we must now identify with Nägeli's micelles, are simply bundles of long chainmolecules, bundles of varying size and degree of perfection of organisation, and probably without any particularly sharp demarcation one from another. In the case of fibres of cellulose and natural silk, when the X-ray data are submitted to detailed analysis and compared with the results of tensile experiments and the findings of organic chemistry, this concept leads further to the decision that the chain-molecules are stereochemically fully extended; but we immediately encounter difficulties when we try to apply these ideas to the study of protein structures other than silk, such as hair, collagen, muscle, etc. The main obstacle, however, is removed by the discovery and interpretation of the X-ray photograph of stretched hair, a photograph which shows that the molecule or complex of hair keratin, when pulled, undergoes a reversible intra-molecular transformation into an elongated stereo-isomer in which the polypeptide chains are analogous in form to those of silk fibroin, that is to say, are fully extended and correspond to the normal polypeptide chains of the chemist. It follows, therefore, that the chains in unstretched hair are in equilibrium in a folded state, so that the mechanism of its extraordinary long-range olasticity is inherent in the keratin molecule itself: by the application of tension in the presence of water the keratin molecule can be stretched to roughly twice its equilibrium length, to which it returns exactly when the tension is removed.

More recent work on this problem indicates now that the 'unit' of the keratin complex is actually a polypeptide sheet or 'grid' in which the mainchains are linked side-to-side by a long series of roughly co-planar cross-linkages formed by the side-chains of the various amino-acid residues incorporated in the structure. The folds in the main-chains of unstretched hair referred to above lie apparently in planes transverse to the sidechains, as one would perhaps rather expect : each grid simply flattens out when pulled in the direction of the main-chains, thereby giving rise to a complex system of stresses and strains which must be the basis of the observed long-range elasticity. From this point of view the elastic properties of keratin are in no way different in principle from those of 
the simpler molecules; the latter, too, are sus. ceptible of distortion within the limits imposed by inter-bond angles, electrostatic attractions, rotation about bonds, and so on, but in keratin the possibilities are so enormously enhanced by the length and mobility of both main-chains and side-chains that at first sight we appear to be dealing with a new phenomenon.

The most beautiful example of this line of reasoning is afforded by feather keratin, which gives an $\mathrm{X}$-ray photograph at the moment unique in crystal analysis. Besides revealing quantitatively and for the first time the truly heroic proportions of a protein molecule, this photograph shows also that the molecule or complex of feather keratin can be stretched continuously and reversibly up to as much as 7 per cent of its equilibrium length! It seems clear, too, that we are again operating with a net- or grid-like system, a molecular device which we may feel sure is common in biological structures, and of which the elastic properties are of fundamental importance for our knowledge of the mechanism of both growth and movement.

The paper presented by Dr. J. B. Speakman on the co-ordination of chemistry and X-ray analysis in fibre research followed admirably on the above account, emphasising as it did once more the extreme fruitfulness of a union of branches which, alone in fields of such bewildering complexity, might well prove barren. There is a pronounced difference in the lateral swelling of wool or hair in weak and strong acids, the former being far more effective. Considerations based on the Donnan equilibrium indicate why this should be so, and the argument is given stereochemical form, so to speak, by the corresponding $\mathrm{X}$-ray photographs, which show how, in hydrochloric acid, for example, the main outlines of the keratin complex are scarcely disturbed, though in quite a dilute solution of chloracetic acid the diffraction pattern is obliterated completely, only to return in all perfection when the acid is removed by washing and drying. It was pointed out. by Speakman how this observation offers a possible means of estimating the size of the grid-like units of keratin suggested by $\mathrm{X}$-ray analysis; for we should be able, from a study of heats of reaction and swelling, to measure the total inter-grid cohesion in the extended form ( $\beta$-keratin) as compared with that in the single-chain protein of silk, to which X-rays have shown the main chains of $\beta$-keratin to be analogous (see above). Experiments to this end are in progress.

The study of the effects of de-aminating animal hairs provides a still more instructive example of the value of $\mathrm{X}$-ray and chemical collaboration. Stretched hair, as is well known, can be 'set' in the elongated form by exposure to steam, and $\mathrm{X}$. rays show that this is due to a re-distribution of cross-linkages in the keratin grid, whereby a new equilibrium configuration is taken up with the main-chains in the extended state. The reversibility of the intra-molecular transformation is thus destroyed by prolonged steaming, and the photograph of $\beta$-keratin persists. The remarkable thing now is that it is found that de-aminated hair has lost this power of 'permanent set', and to an extent depending on the degree of de-amination. The change can be followed throughout by means of X-rays, which show at once whether the $\beta$-photograph is 'set' after a given amount of de-amination and steaming of the fibre in the stretched state.

The experiments are a most valuable contribution to our knowledge of the chemistry of keratin, and therefore of all proteins, for we may now feel confident not only that the process of 'setting' wool and hair involves the $-\mathrm{NH}_{2}$ groups of the basic side-chains, but also that the contractile power of keratin is by no means destroyed-rather is its range extended--on their removal. In view of the theory of K. H. Meyer that the contractile power of muscle arises from attractions between basic and acidic side-chains of one and the same main-chain, it is clear that this discovery may have far-reaching implications.

The botanists were offered an elegant piece of structure analysis by Dr. R. D. Preston who, continuing the work of Astbury, Marwick and Bernal which brought to light that the cell-wall of the alga, Valonia ventricosa, is constructed of two sets of cellulose chains crossing according to some regular plan at an angle near a right angle, described the present state of an X-ray exploration of the whole of the wall of a single complete cell. Since a normal photograph taken at any point of the wall gives the two cellulose directions at that point, the method adopted is to follow up one of the directions exactly as one follows lines of force with a small compass needle, the results being afterwards plotted both on the cell-wall itself and on a large-scale model made from a bladder.

The investigation is necessarily a prolonged one with the modest apparatus available, but already the findings are of a highly intriguing character. They show to date that the molecular structure of this cellulose balloon is built up in spiral fashion, exactly as are the cellulose fibres ramie, cotton, etc. The completion of the investigation will no doubt be eagerly awaited, for there is a widespread interest in the structure and metabolism of Valonia. The single cells of this alga are the first to be explored in detail by $\mathrm{X}$-ray methods, and the discovery that it shares with the fibres a spiral architecture must be of deep significance for the problem of the mechanism of growth.

The mechanism of growth was also indirectly the subject of a fascinating contribution by $\mathrm{Mr}$. $J$. Thewlis, who showed how X-rays have revealed the arrangement of the apatite crystals which constitute the enamel of teeth. Tooth enamel, like so many other biological structures, is of a fibrous nature, the hexagonal axis of the apatite crystals being the fibre axis. In human enamel there are two sets of fibres, one with the fibre axis inclined at about $20^{\circ}$ to the normal to the tooth surface and on the same side as the tip, the other at about $10^{\circ}$ and on the opposite side to the 
tip. In dog's enamel the fibre axis is at right angles to the surface of the tooth. Variations in the perfection of fibre orientation are observed, and three kinds of enamel can be distinguished. In human teeth it is found that one kind is associated with clinically immune teeth, and the other two with clinically susceptible teeth. Here again the verdict of $\mathrm{X}$-ray analysis must ultimately prove of fundamental importance in the study of living things, and it is to be hoped that this most promising field of investigation will soon be extended so as to take in the effects of the action of vitamins.
The biological implications of recent advances in the X-ray analysis of protein fibres were again dealt with by Mr. W. T. Astbury at the discussion on the structure of protoplasm. No doubt some of the zoologists present were not a little shocked at such heresy, but nevertheless the message of X-rays seems clear enough. The proteins are infinitely variable and adjustable molecular patterns, exquisitely sensitive to changes in physical and chemical environment, and capable of functioning not only as enzymes but also as the material embodiment of the genes. Surely they are no other than the very patterns of life! W. T.A.

\section{News and Views}

\section{Honorary Fellows of the Physical Society}

THe list of honorary fellows of the Physical Society was lengthened on October 6 by the addition of the names of Profs. F. Paschen, A. Sommerfeld and R. W. Wood. Friedrich Paschen, director of the Reichsanstalt until last year, was born in 1865, and has crowded an immense amount of fundamental work into the intervening years. 'Paschen's law', governing the sparking potential between electrodes, is as familiar as the Paschen series in the line spectrum of hydrogen, whilst the Paschen galvanometer (now nearly forty years old) is still probably the most sensitive yet constructed. These three examples remind us of his versatility, but it has to be remem. bered that he also measured the heat evolution of radium, investigated the Doppler effect in canal rays, measured a great number of wave-lengths in the ultra-violet and infra-red and, above all, was one of those who established experimentally the form of the spectral distribution curve for a black body.

Arnowd Sommerfend, best known by that encyclopædic summary of the old quantum mechanies, "Atombau und Spektrallinien", is three years the junior of Paschen. He occupies a chair at the University of Munich, and his contributions to knowledge are all mathematical. He has contributed to subjects so diverse as the calculation of the A.c. resistance of coils, and the theory of optical dispersion. In the early days of wireless, he, like most of the mathematical physicists of the time, took part in the discussion on how the waves managed to follow the curvature of the earth. More familiar to students is his mathematical formulation of the intensity due to a wave, regarded as a summation of the effects due to Huygens' wavelets, though his fame rests chiefly on the refinements which he applied to Bohr's theory of the atom, such as the taking into account of elliptical orbits, and the application of a relativity correction to the equations of motion of an electron, thus correctly accounting for the fine structure of the hydrogen lines.

THE third of this distinguished trio, Prof. R. W. Wood, was borm in the same year as Sommerfeld, and is, like him, a foreign member of the Royal Society. Professor at the John Hopkins University, scene of the 'Baltimore Lectures', it is fitting that his interests should have centred around wave theory, first in optics and more recently in supersonics. His experimental genius is universally acknowledged, and his textbook on "Physical Optics" - as it must be with such an author-is not only full of facts in all branches of its subject, but also contains a wealth of practical advice. Light filters, the construction of zone plates, the making of a prism with an angle of a few minutes (or was it seconds ?) of arc, all received the same careful attention. Perhaps his greatest single discovery was that of resonance radiation. Great as it was in itself, it came at the right time to help the theoretical physicists to work out the conception of energy levels and quantum transitions in the atom.

\section{Guthrie and the Physical Society}

ON October 15 occurs the centenary of the birth of Frederick Guthrie, through whose initiative the Physical Society of London was founded. Born in Bayswater, London, Guthrie was the son of a Bond Street tailor, and as a boy had as tutor the chemist Henry Watts (1815-1884), to whom no doubt he owed his early devotion to science. $\mathrm{He}$ was sent to University College School, and afterwards entered University College, where Watts was an assistant professor, and studied chemistry under Graham and Williamson, and mathematics under De Morgan. From London, at the age of twenty-one, he went to Germany, working under Bunsen at Heidelberg and under Kolbe at Marburg. On his return to England he was successively assistant to Frankland at Owens College and to Lyon Playfair at Edinburgh. In 1861 he was appointed professor of chemistry and physics at the Royal College, Mauritius, where he had as his colleague Walter Besant, the novelist. In 1869 he became professor of physics at the Normal College of Science, South Kensington, and this post he held until his death.

GUTHRIE's early published papers related to chemistry, but in Mauritius he turned to the study of physical problems and afterwards published memoirs and textbooks on heat, magnetism and 